\title{
Predicting low-frequency radio fluxes of known extrasolar planets ${ }^{\star \star \star}$
}

\author{
J.-M. Grießmeier ${ }^{1}$, P. Zarka ${ }^{1}$, and H. Spreeuw ${ }^{2}$ \\ 1 LESIA, Observatoire de Paris, CNRS, UPMC, Université Paris Diderot; 5 Place Jules Janssen, 92190 Meudon, France \\ e-mail: [jean-mathias.griessmeier;philippe.zarka]@obspm. fr \\ 2 Astronomical Institute "Anton Pannekoek", Kruislaan 403, 1098 SJ Amsterdam, The Netherlands \\ e-mail: hspreeuw@science.uva.nl \\ Received 3 March 2007 / Accepted 18 August 2007
}

\section{ABSTRACT}

\begin{abstract}
Context. Close-in giant extrasolar planets ("Hot Jupiters") are believed to be strong emitters in the decametric radio range.
Aims. We present the expected characteristics of the low-frequency magnetospheric radio emission of all currently known extrasolar planets, including the maximum emission frequency and the expected radio flux. We also discuss the escape of exoplanetary radio emission from the vicinity of its source, which imposes additional constraints on detectability.

Methods. We compare the different predictions obtained with all four existing analytical models for all currently known exoplanets. We also take care to use realistic values for all input parameters.

Results. The four different models for planetary radio emission lead to very different results. The largest fluxes are found for the magnetic energy model, followed by the CME model and the kinetic energy model (for which our results are found to be much less optimistic than those of previous studies). The unipolar interaction model does not predict any observable emission for the present exoplanet census. We also give estimates for the planetary magnetic dipole moment of all currently known extrasolar planets, which will be useful for other studies.

Conclusions. Our results show that observations of exoplanetary radio emission are feasible, but that the number of promising targets is not very high. The catalog of targets will be particularly useful for current and future radio observation campaigns (e.g. with the VLA, GMRT, UTR-2 and with LOFAR).
\end{abstract}

Key words. radiation mechanisms: non-thermal - catalogs - plasmas - planets and satellites general

\section{Introduction}

In the solar system, all strongly magnetised planets are known to be intense nonthermal radio emitters. For a certain class of extrasolar planets (the so-called Hot Jupiters), an analogous, but much more intense radio emission is expected. In the recent past, such exoplanetary radio emission has become an active field of research, with both theoretical studies and ongoing observation campaigns.

Recent theoretical studies have shown that a large variety of effects have to be considered, e.g. kinetic, magnetic and unipolar interaction between the star (or the stellar wind) and the planet, the influence of the stellar age, the potential role of stellar CMEs, and the influence of different stellar wind models. So far, there is no single publication in which all of these aspects are put together and where the different interaction models are compared extensively. We also discuss the escape of exoplanetary radio emission from its planetary system, which depends on the local stellar wind parameters. As will be shown, this is an additional constraint for detectability, making the emission from several planets impossible to observe.

The first observation attempts go back at least to Yantis et al. (1977). At the beginning, such observations were

\footnotetext{
* Appendices A and B are only available in electronic form at http: //www . aanda. org

$\star \star$ Table 1 is only available in electronic form at the CDS via anonymous ftp to cdsarc.u-strasbg.fr $(130.79 .128 .5)$ or via http://cdsweb.u-strasbg.fr/cgi-bin/qcat?J/A+A/475/359
}

necessarily unguided ones, as exoplanets had not yet been discovered. Later observation campaigns concentrated on known exoplanetary systems. So far, no detection has been achieved. A list and a comparison of past observation attempts can be found elsewhere (Grießmeier et al. 2006a). Concerning ongoing and future observations, studies are performed or planned at the VLA (Lazio et al. 2004), GMRT (Majid et al. 2006; Winterhalter et al. 2006), UTR2 (Ryabov et al. 2004), and at LOFAR (Farrell et al. 2004). To support these observations and increase their efficiency, it is important to identify the most promising targets.

The target selection for radio observations is based on theoretical estimates which aim at the prediction of the main characteristics of the exoplanetary radio emission. The two most important characteristics are the maximum frequency of the emission and the expected radio flux. The first predictive studies (e.g. Zarka et al. 1997; Farrell et al. 1999) concentrated on only a few exoplanets. A first catalog containing estimations for radio emission of a large number of exoplanets was presented by Lazio et al. (2004). This catalog included 118 planets (i.e. those known as of 2003, July 1) and considered radio emission energised by the kinetic energy of the stellar wind (i.e. the kinetic model, see below). Here, we present a much larger list of targets (i.e. 197 exoplanets found by radial velocity and/or transit searches as of 2007, January 13, taken from http: //exoplanet.eu/), and compare the results obtained by all four currently existing interaction models, not all of which were known at the time of the previous overview study. As a byproduct of the radio flux calculation, we obtain estimates for 
the planetary magnetic dipole moment of all currently known extrasolar planets. These values will be useful for other studies as, e.g., star-planet interaction or atmospheric shielding.

To demonstrate which stellar and planetary parameters are required for the estimation of exoplanetary radio emission, some theoretical results are briefly reviewed (Sect. 2). Then, the sources for the different parameters (and their default values for the case where no measurements are available) are presented (Sect. 3). In Sect. 4, we present our estimations for exoplanetary radio emission. This section also includes estimates for planetary magnetic dipole moments. Section 5 closes with a few concluding remarks.

\section{Exoplanetary radio emission theory}

\subsection{Expected radio flux}

In principle, there are four different types of interaction between a planetary obstacle and the ambient stellar wind, as both the stellar wind and the planet can either be magnetised or unmagnetised. Zarka (2007, Table 1) show that for three of these four possible situations intense nonthermal radio emission is possible. Only in the case of an unmagnetised stellar wind interacting with an unmagnetised body no intense radio emission is possible.

In those cases where strong emission is possible, the expected radio flux depends on the source of available energy. In the last years, four different energy sources were suggested: a) in the first model, the input power $P_{\text {input }}$ into the magnetosphere is assumed to be proportional to the total kinetic energy flux of the solar wind protons impacting on the magnetopause (Desch \& Kaiser 1984; Zarka et al. 1997; Farrell et al. 1999; Zarka et al. 2001b; Farrell et al. 2004; Lazio et al. 2004; Stevens 2005; Grießmeier et al. 2005, 2006b, 2007); b) similarly, the input power $P_{\text {input }}$ into the magnetosphere can be assumed to be proportional to the magnetic energy flux or electromagnetic Poynting flux of the interplanetary magnetic field (Zarka et al. 2001b; Farrell et al. 2004; Zarka 2004, 2006, 2007). From the data obtained in the solar system, it is not possible to distinguish which of these models is more appropriate (the constants of proportionality implied in the relations given below are not well known, see Zarka et al. 2001b), so that both models have to be considered; c) for unmagnetised or weakly magnetised planets, one may apply the unipolar interaction model. In this model, the star-planet system can be seen as a giant analog to the Jupiter-Io system (Zarka et al. 2001b; Zarka 2004, 2006, 2007). Technically, this model is very similar to the magnetic energy model, but the source location is very different: whereas in the kinetic and in the magnetic model, the emission is generated near the planet, in the unipolar interaction case a large-scale current system is generated and the radio emission is generated in the stellar wind between the star and the planet. Thus, the emission can originate from a location close to the stellar surface, close to the planetary surface, or at any point between the two. This is possible in those cases where the solar wind speed is lower than the Alfvén velocity (i.e. for close-in planets, see e.g. Preusse et al. 2005). Previous studied have indicated that this emission is unlikely to be detectable, except for stars with an extremely strong magnetic field (Zarka et al. 2001b; Zarka 2004, 2006, 2007). Nevertheless, we will check whether this type of emission is possible for the known exoplanets; d) the fourth possible energy source is based on the fact that closein exoplanets are expected to be subject to frequent and violent stellar eruptions (Khodachenko et al. 2007b) similar to solar coronal mass ejections (CMEs). As a variant to the kinetic energy model, the $C M E$ model assumes that the energy for the most intense planetary radio emission is provided by CMEs. During periods of such CME-driven radio activity, considerably higher radio flux levels can be achieved than during quiet stellar conditions (Grießmeier et al. 2006b, 2007). For this reason, this model is treated separately.

For the kinetic energy case, the input power was first derived by Desch \& Kaiser (1984), who found that it is given by

$P_{\text {input,kin }} \propto n v_{\mathrm{eff}}^{3} R_{\mathrm{s}}^{2}$.

In Eq. (1), $n$ is the stellar wind density at the planetary orbit, $v_{\text {eff }}$ is the velocity of the stellar wind in the reference frame of the planet (i.e. including the aberration due to the orbital velocity of the planet, which is not negligible for close-in planets), and $R_{\mathrm{s}}$ denotes the magnetospheric standoff distance.

The magnetic energy case was first discussed by Zarka et al. (2001b). Here, the input power is given by

$P_{\text {input,mag }} \propto v_{\text {eff }} B_{\perp}^{2} R_{\mathrm{s}}^{2}$.

In Eq. (2), $v_{\text {eff }}$ is the velocity of the stellar wind in the reference frame of the planet, $B_{\perp}$ is the component of the interplanetary magnetic field (IMF) perpendicular to the stellar wind flow in the reference frame of the planet, and $R_{\mathrm{s}}$ denotes the magnetospheric standoff distance.

For the unipolar interaction case (Zarka et al. 2001b), the input power is given by

$P_{\text {input,unipolar }} \propto v_{\text {eff }} B_{\perp}^{2} R_{\text {ion }}^{2}$

Eq. (3) is identical to Eq. (2), except that the obstacle is not the planetary magnetosphere, but its ionosphere, so that $R_{\mathrm{S}}$ is replaced by $R_{\text {ion }}$, the radius of the planetary ionosphere.

$C M E$-driven radio emission was first calculated by Grießmeier et al. (2006b). In that case, the input power is given by

$P_{\text {input,kin,CME }} \propto n_{\mathrm{CME}} v_{\mathrm{eff}, \mathrm{CME}}^{3} R_{\mathrm{s}}^{2}$.

Equation (4) is identical to Eq. (1), except that the stellar wind density and velocity are replaced by the corresponding values encountered by the planet during a CME.

A certain fraction $\epsilon$ of the input power $P_{\text {input }}$ given by Eqs. (1), (2), (3) or (4) is thought to be dissipated within the magnetosphere:

$P_{d}=\epsilon P_{\text {input }}$.

Observational evidence suggests that the amount of power emitted by radio waves $P_{\text {rad }}$ is roughly proportional to the power input $P_{\text {input }}$ (see, e.g. Zarka 2007, Fig. 6). This can be written as:

$P_{\text {radio }}=\eta_{\text {radio }} P_{d}=\eta_{\text {radio }} \epsilon P_{\text {input }}$.

As $P_{d}$ cannot be measured directly, one correlates the observed values of $P_{\text {radio }}$ with the calculated (model dependent) values of $P_{\text {input }}$. Thus, one replaces $P_{\text {input }}$ by $P_{\text {radio }}$ on the left-hand side of the proportionalities given by $(1)-(4)$. The proportionality constant is determined by comparison with Jupiter. The analysis of the jovian radio emission allows to define three values for the typical radio spectrum: (a) the power during average conditions, (b) the average power during periods of high activity, and (c) the peak power (Zarka et al. 2004). In this work, we will use the average power during periods of high activity as a reference value for all four cases, with $P_{\text {radio,J }}=2.1 \times 10^{11} \mathrm{~W}$. 
The radio flux $\Phi$ seen by an observer at a distance $s$ from the emitter is related to the emitted radio power $P_{\text {radio }}$ by (Grießmeier et al. 2007):

$\Phi=\frac{P_{\text {radio }}}{\Omega s^{2} \Delta f}=\frac{4 \pi^{2} m_{\mathrm{e}} R_{\mathrm{p}}^{3} P_{\text {radio }}}{e \mu_{0} \Omega s^{2} \mathcal{M}}$.

Here, $\Omega$ is the solid angle of the beam of the emitted radiation ( $\Omega=1.6 \mathrm{sr}$, see Zarka et al. 2004), and $\Delta f$ is the bandwidth of the emission. We use $\Delta f=f_{\mathrm{c}}^{\max }$ (Grießmeier et al. 2007), where $f_{\mathrm{c}}^{\max }$ is the maximum cyclotron frequency. Depending on the model, $P_{\text {radio }}$ is given by Eqs. (1), (2), (3) or (4). The maximum cyclotron frequency $f_{\mathrm{c}}^{\max }$ is determined by the maximum magnetic field strength $B_{\mathrm{p}}^{\max }$ close to the polar cloud tops (Farrell et al. 1999):

$f_{\mathrm{c}}^{\max }=\frac{e B_{\mathrm{p}}^{\max }}{2 \pi m_{\mathrm{e}}}=\frac{e \mu_{0} \mathcal{M}}{4 \pi^{2} m_{\mathrm{e}} R_{\mathrm{p}}^{3}} \approx 24 \mathrm{MHz} \frac{\widetilde{\mathcal{M}}}{{\widetilde{R_{\mathrm{p}}}}^{3}}$.

Here, $m_{\mathrm{e}}$ and $e$ are the electron mass and charge, $R_{\mathrm{p}}$ is the planetary radius, $\mu_{0}$ is the magnetic permeability of the vacuum, and $\mathcal{M}$ is the planetary magnetic dipole moment. $\widetilde{\mathcal{M}}$ and $\widetilde{R_{\mathrm{p}}}$ denote the planetary magnetic moment and its radius relative to the respective value for Jupiter, e.g. $\widetilde{\mathcal{M}}=\mathcal{M} / \mathcal{M}_{\mathrm{J}}$, with $\mathcal{M}_{\mathrm{J}}=1.56 \times 10^{27} \mathrm{~A} \mathrm{~m}^{2}$ (Cain et al. 1995) and $R_{\mathrm{J}}=71492 \mathrm{~km}$.

The radio flux expected for the four different models according to Eqs. (1) to (7) and the maximum emission frequency according to (8) are calculated in Sect. 4 for all known exoplanets.

\subsection{Escape of radio emission}

To allow an observation of exoplanetary radio emission, it is not sufficient to have a high enough emission power at the source and emission in an observable frequency range. As an additional requirement, it has to be checked that the emission can propagate from the source to the observer. This is not the case if the emission is absorbed or trapped (e.g. in the stellar wind in the vicinity of the radio-source), which happens whenever the plasma frequency

$f_{\text {plasma }}=\frac{1}{2 \pi} \sqrt{\frac{n e^{2}}{\epsilon_{0} m_{\mathrm{e}}}}$

is higher than the emission frequency at any point between the source and the observer. Thus, the condition of observability is

$f_{\text {plasma }}^{\max }<f_{\mathrm{c}}^{\max }$,

where $f_{\mathrm{c}}^{\max }$ is taken at the radio source (e.g. the planet), whereas $f_{\text {plasma }}^{\text {max }}$ is evaluated along the line of sight. As the density of the electrons in the stellar wind $n$ decreases with the distance to the star, this condition is more restrictive at the orbital distance of the planet than further out. Thus, it is sufficient to check whether condition (10) is satisfied at the location of the radiosource (i.e. for $n=n(d)$, where $d$ is the distance from the star to the radio-source). In that case, the emission can escape from the planetary system and reach distant observers. In Sect. 4, condition (10) is checked for all known exoplanets at their orbital distance.

Note however that, depending on the line of sight, not all observers will be able to see the planetary emission at all times. For example, the observation of a secondary transit implies that the line of sight passes very close to the planetary host star, where the plasma density is much higher. For this reason, some parts of the orbit may be unobservable even for planets for which Eq. (10) is satisfied.

\subsection{Radiation emission in the unipolar interaction model}

An additional constraint arises because certain conditions are necessary for the generation of radio emission. Planetary radio emission is caused by the cyclotron maser instability (CMI). This mechanism is only efficient in regions where the ratio between the electron plasma frequency and the electron cyclotron frequency is small enough. This condition can be written as

$\frac{f_{\text {plasma }}}{f_{\mathrm{c}}} \lesssim 0.4$,

where the electron cyclotron frequency $f_{\mathrm{c}}$ is defined by the local magnetic field

$f_{\mathrm{c}}=\frac{e B}{2 \pi m_{\mathrm{e}}}$

and $f_{\text {plasma }}$ is given by Eq. (9). Observations seem to favor a critical frequency ratio close to the 0.1 , while theoretical work supports a critical frequency ratio close to 0.4 (Le Quéau et al. 1985; Hilgers 1992; Zarka et al. 2001a). Fundamental O mode or second harmonic $\mathrm{O}$ and $\mathrm{X}$ mode emission are possible also for larger frequency ratios, but are much less efficient (Treumann 2000; Zarka 2007). To avoid ruling out potential emission, we use the largest possible frequency ratio, i.e. $\frac{f_{\text {plasma }}}{f_{\mathrm{c}}} \leq 0.4$.

The condition imposed by Eq. (11) has to be fulfilled for any of the four models presented in Sect. 2.1. For the three models where the radio emission is generated directly in the planetary magnetosphere, $n$ decreases much faster with distance to the planetary surface than $B$, so that Eq. (11) can always be fulfilled. For the unipolar interaction model, the emission takes place in the stellar wind, and the electron density $n$ can be obtained from the model of the stellar wind. In this case, it is not a priori clear whether and where radio emission is possible. It could be generated anywhere between the star and the planet. In Sect. 4, we will check separately for each planetary system whether unipolar interaction satisfying Eq. (11) is possible at any location between the stellar surface and the planetary orbit.

\section{Required parameters}

In the previous section, it has been shown that the detectability of planetary radio emission depends on a few planetary parameters:

- the planetary radius $R_{\mathrm{p}}$;

- the planetary magnetic moment $\mathcal{M}$;

- the size of the planetary magnetosphere $R_{\mathrm{s}}$;

- the size of the planetary ionosphere $R_{\text {ion }}$;

- the stellar wind density $n$ and its velocity $v_{\text {eff }}$;

- the stellar magnetic field (IMF) $B_{\perp}$ perpendicular to the stellar wind flow in the frame of the planet;

- the distance of the stellar system (to an earth-based observer) $s$;

- the solid angle of the beam of the emitted radiation $\Omega$.

The models used to infer the missing stellar and planetary quantities require the knowledge of a few additional planetary parameters. These are the following:

- the planetary mass $M_{\mathrm{p}}$;

- the planetary radius $R_{\mathrm{p}}$;

- its orbital distance $d$;

- the planetary rotation rate $\omega$;

- the stellar magnetic field (IMF) components $B_{r}, B_{\phi}$;

- the stellar mass $M_{\star}$; 
- the stellar radius $R_{\star}$;

- the stellar age $t_{\star}$.

In this section, we briefly describe how these each of these quantities can be obtained.

\subsection{Basic planetary parameters}

As a first step, basic planetary characteristics have to be evaluated:

- $d, \omega_{\text {orbit }}$ and $s$ are directly taken from the Extrasolar Planets Encyclopaedia at http://exoplanet.eu, as well as the observed mass $M_{\mathrm{obs}}$ and the orbital eccentricity $e$. Note that in most cases the observed mass is the "projected mass" of the planet, i.e. $M_{\mathrm{obs}}=M_{\mathrm{p}} \sin i$, where $i$ is the angle of inclination of the planetary orbit with respect to the observer.

- For eccentric planets, we calculate the periastron from the semi-major axis $d$ and the orbital eccentricity $e: d_{\min }=$ $d /(1-e)$. Thus, the results for planetary radio emission apply to the periastron.

- For most exoplanets, the planetary mass is not precisely known. Instead, usually only the projected mass $M_{\mathrm{p}} \sin i$ is accessible to measurements, where $i$ is the inclination of the planetary orbit with respect to the observer. This projected mass is taken from http: //exoplanet . eu and converted to the median value of the mass: $\operatorname{median}\left(M_{\mathrm{p}}\right)=$ $M_{\mathrm{obs}} \cdot \operatorname{median}\left(\frac{1}{\sin i}\right)=\sqrt{4 / 3} \cdot M_{\mathrm{obs}} \approx 1.15 M_{\mathrm{obs}}$.

- For transiting planets, $R_{\mathrm{p}}$ is taken from original publications. For non-transiting planets, the planetary radius is $R_{\mathrm{p}}$ not known. In this case, we estimate the planetary radius based on its mass $M_{\mathrm{p}}$ and orbital distance $d$, as explained in Appendix A. The radius of a "cold" planet of mass $M_{\mathrm{p}}$ is given by

$$
R_{\mathrm{p}}(d=\infty)=\frac{\left(\alpha M_{\mathrm{p}}\right)^{1 / 3}}{1+\left(\frac{M_{\mathrm{p}}}{M_{\max }}\right)^{2 / 3}} \approx 1.47 R_{\mathrm{J}} \frac{{\widetilde{M_{\mathrm{p}}}}^{1 / 3}}{1+\left(\frac{\widetilde{M_{\mathrm{p}}}}{\widetilde{M}_{\max }}\right)^{2 / 3}}
$$

with $\alpha=6.1 \times 10^{-4} \mathrm{~m}^{3} \mathrm{~kg}^{-1}$ (for a planet with the same composition as Jupiter) and $M_{\max }=3.16 M_{\mathrm{J}}$. Again, $\widetilde{M}_{\mathrm{p}}$ and $\widetilde{M_{\max }}$ denote values relative to the respective value for Jupiter (using $M_{\mathrm{J}}=1.9 \times 10^{27} \mathrm{~kg}$ ). The radius of an irradiated planet is then given by

$$
\frac{R_{\mathrm{p}}(d)}{R_{\mathrm{p}}(d=\infty)}=\frac{\widetilde{R_{\mathrm{p}}}(d)}{\widetilde{R_{\mathrm{p}}}(d=\infty)}=\left[1+0.05\left(\frac{T_{\mathrm{eq}}}{T_{0}}\right)^{\gamma}\right]
$$

where $T_{\text {eq }}$ is the equilibrium temperature of the planetary surface. The coefficients $T_{0}$ and $\gamma$ depend on the planetary mass (see Appendix A).

\subsection{Stellar wind model}

The stellar wind density $n$ and velocity $v_{\text {eff }}$ encountered by a planet are key parameters defining the size of the magnetosphere and thus the energy flux available to create planetary radio emission. As these stellar wind parameters strongly depend on the stellar age, the expected radio flux is a function of the estimated age of the exoplanetary host star (Stevens 2005; Grießmeier et al. 2005). At the same time it is known that at close distances the stellar wind velocity has not yet reached the value it has at larger orbital distances. For this reason, a distance-dependent stellar wind models has to be used to avoid overestimating the expected planetary radio flux (Grießmeier 2006; Grießmeier et al. 2007).

It was shown (Grießmeier et al. 2007) that for stellar ages $>0.7 \mathrm{Gyr}$, the radial dependence of the stellar wind properties can be described by the stellar wind model of Parker (1958), and that the more complex model of Weber \& Davis (1967) is not required. In the Parker model, the interplay between stellar gravitation and pressure gradients leads to a supersonic gas flow for sufficiently large substellar distances $d$. The free parameters are the coronal temperature and the stellar mass loss. They are indirectly chosen by setting the stellar wind conditions at $1 \mathrm{AU}$. More details on the model can be found elsewhere (e.g. Mann et al. 1999; Preusse 2006; Grießmeier 2006).

The dependence of the stellar wind density $n$ and velocity and $v_{\text {eff }}$ on the age of the stellar system is based on observations of astrospheric absorption features of stars with different ages. In the region between the astropause and the astrospheric bow shock (analogs to the heliopause and the heliospheric bow shock of the solar system), the partially ionized local interstellar medium (LISM) is heated and compressed. Through charge exchange processes, a population of neutral hydrogen atoms with high temperature is created. The characteristic $\operatorname{Ly} \alpha$ absorption (at $1216 \AA$ ) of this population was detectable with the highresolution observations obtained by the Hubble Space Telescope (HST). The amount of absorption depends on the size of the astrosphere, which is a function of the stellar wind characteristics. Comparing the measured absorption to that calculated by hydrodynamic codes, these measurements allowed the first empirical estimation of the evolution of the stellar mass loss rate as a function of stellar age (Wood et al. 2002; Wood 2004; Wood et al. 2005). It should be noted, however, that the resulting estimates are only valid for stellar ages $\geq 0.7$ Gyr (Wood et al. 2005). From these observations, (Wood et al. 2005) calculate the agedependent density of the stellar wind under the assumption of an age-independent stellar wind velocity. This leads to strongly overestimated stellar wind densities, especially for young stars (Grießmeier et al. 2005; Holzwarth \& Jardine 2007). For this reason, we combine these results with the model for the agedependence of the stellar wind velocity of Newkirk (1980). One obtains (Grießmeier et al. 2007):

$v(1 \mathrm{AU}, t)=v_{0}\left(1+\frac{t}{\tau}\right)^{-0.43}$.

The particle density can be determined to be

$n(1 \mathrm{AU}, t)=n_{0}\left(1+\frac{t}{\tau}\right)^{-1.86 \pm 0.6}$,

with $v_{0}=3971 \mathrm{~km} \mathrm{~s}^{-1}, n_{0}=1.04 \times 10^{11} \mathrm{~m}^{-3}$ and $\tau=2.56 \times$ $10^{7} \mathrm{yr}$.

For planets at small orbital distances, the keplerian velocity of the planet moving around its star becomes comparable to the radial stellar wind velocity. Thus, the interaction of the stellar wind with the planetary magnetosphere should be calculated using the effective velocity of the stellar wind plasma relative to the planet, which takes into account this "aberration effect" (Zarka et al. 2001b). For the small orbital distances relevant for Hot Jupiters, the planetary orbits are circular because of tidal dissipation (Goldreich \& Soter 1966; Dobbs-Dixon et al. 2004; Halbwachs et al. 2005). For circular orbits, the orbital velocity $v_{\text {orbit }}$ is perpendicular to the stellar wind velocity $v$, and its value is given by Kepler's law. In the reference frame of the planet, the stellar wind velocity then is given by

$v_{\text {eff }}=\sqrt{v_{\text {orbit }}^{2}+v^{2}}$. 
Finally, for the magnetic energy case, the interplanetary magnetic field $\left(B_{r}, B_{\phi}\right)$ is required. At $1 \mathrm{AU}$, the average field strength of the interplanetary magnetic field is $B_{\mathrm{imf}} \approx 3.5 \mathrm{nT}$ (Mariani \& Neubauer 1990; Prölss 2004). According to the Parker stellar wind model (Parker 1958), the radial component of the interplanetary magnetic field decreases as

$B_{\text {imf }, r}(d)=B_{r, 0}\left(\frac{d}{d_{0}}\right)^{-2}$.

This was later confirmed by Helios measurements. One finds $B_{r, 0} \approx 2.6 \mathrm{nT}$ and $d_{0}=1 \mathrm{AU}$ (Mariani \& Neubauer 1990; Prölss 2004). At the same time, the azimuthal component $B_{\mathrm{imf}, \varphi}$ behaves as

$B_{\text {imf }, \varphi}(d)=B_{\varphi, 0}\left(\frac{d}{d_{0}}\right)^{-1}$,

with $B_{\varphi, 0} \approx 2.4 \mathrm{nT}$ (Mariani \& Neubauer 1990; Prölss 2004). The average value of $B_{\mathrm{imf}, \theta}$ vanishes $\left(B_{\mathrm{imf}, \theta} \approx 0\right)$. From $B_{\mathrm{imf}, r}(d)$ and $B_{\text {imf }, \varphi}(d)$, the stellar magnetic field (IMF) $B_{\perp}(d)$ perpendicular to the stellar wind flow in the frame of the planet can be calculated (Zarka 2007):

$B_{\perp}=\sqrt{B_{\mathrm{imf}, r}^{2}+B_{\mathrm{imf}, \varphi}^{2}}|\sin (\alpha-\beta)|$

with

$\alpha=\arctan \left(\frac{B_{\mathrm{imf}, \varphi}}{B_{\mathrm{imf}, r}}\right)$

and

$\beta=\arctan \left(\frac{v_{\text {orbit }}}{v}\right)$.

We obtain the stellar magnetic field $B_{\star}$ relative to the solar magnetic field $B_{\odot}$ under the assumption that it is inversely proportional to the rotation period $P_{\star}$ (Collier Cameron \& Jianke 1994; Grießmeier et al. 2007):

$\frac{B_{\star}}{B_{\odot}}=\frac{P_{\odot}}{P_{\star}}$,

where we use $P_{\odot}=25.5 \mathrm{~d}$ and take $B_{\odot}=1.435 \times 10^{-4} \mathrm{~T}$ as the reference magnetic field strength at the solar surface (Preusse et al. 2005). The stellar rotation period $P_{\star}$ is calculated from the stellar age $t$ (Newkirk 1980):

$P_{\star} \propto\left(1+\frac{t}{\tau}\right)^{0.7}$

where the time constant $\tau$ is given by $\tau=2.56 \times 10^{7} \mathrm{yr}$ (calculated from Newkirk 1980).

Note that first measurements of stellar magnetic fields for planet-hosting stars are just becoming available from the spectropolarimeter ESPaDOnS (Catala et al. 2007). This will lead to an improved understanding of stellar magnetic fields, making more accurate models possible in the future.

\subsection{Stellar CME model}

For the CME-driven radio emission, the stellar wind parameters $n$ and $v_{\text {eff }}$ are effectively replaced by the corresponding CME parameters $n_{\mathrm{CME}}$ and $v_{\text {eff,CME}}$, potentially leading to much more intense radio emission than those driven by the kinetic energy of the steady stellar wind (Grießmeier 2006; Grießmeier et al. 2006b, 2007).
These CME parameters are estimated by Khodachenko et al. (2007b), who combine in-situ measurements near the sun (e.g. by Helios) with remote solar observation by SoHO. Two interpolated limiting cases are given, denoted as weak and strong CMEs, respectively. These two classes have a different dependence of the average density on the distance to the star $d$. In the following, these quantities will be labeled $n_{\mathrm{CME}}^{w}(d)$ and $n_{\mathrm{CME}}^{s}(d)$, respectively.

For weak CMEs, the density $n_{\mathrm{CME}}^{w}(d)$ behaves as

$n_{\mathrm{CME}}^{w}(d)=n_{\mathrm{CME}, 0}^{w}\left(d / d_{0}\right)^{-2.3}$

where the density at $d_{0}=1 \mathrm{AU}$ is given by $n_{\mathrm{CME}, 0}^{w}=n_{\mathrm{CME}}^{w}(d=$ $\left.d_{0}\right)=4.9 \times 10^{6} \mathrm{~m}^{-3}$.

For strong CMEs, Khodachenko et al. (2007b) find

$n_{\mathrm{CME}}^{s}(d)=n_{\mathrm{CME}, 0}^{s}\left(d / d_{0}\right)^{-3.0}$

with $n_{\mathrm{CME}, 0}^{s}=n_{\mathrm{CME}}^{s}\left(d=d_{0}\right)=7.1 \times 10^{6} \mathrm{~m}^{-3}$, and $d_{0}=1 \mathrm{AU}$.

As far as the CME velocity is concerned, one has to note that individual CMEs have very different velocities. However, the average $\mathrm{CME}$ velocity $v$ is approximately independent of the subsolar distance, and is similar for both types of CMEs:

$v_{\mathrm{CME}}^{w}=v_{\mathrm{CME}}^{s}=v_{\mathrm{CME}} \approx 500 \mathrm{~km} \mathrm{~s}^{-1}$.

Similarly to the steady stellar wind the CME velocity given by Eq. (27) has to be corrected for the orbital motion of the planet:

$v_{\mathrm{eff}, \mathrm{CME}}=\sqrt{\frac{M_{\star} G}{d}+v_{\mathrm{CME}}^{2}}$.

In addition to the density and the velocity, the temperature of the plasma in a coronal mass ejection is required for the calculation of the size of the magnetosphere. According to Khodachenko et al. $(2007 \mathrm{~b}, \mathrm{a})$, the front region of a CME consists of hot, coronal material $(T \approx 2 \mathrm{MK})$. This region may either be followed by relatively cool prominence material $(T \approx 8000 \mathrm{~K})$, or by hot flare material $(T \approx 10 \mathrm{MK})$. In the following, the temperature of the leading region of the $\mathrm{CME}$ will be used, i.e. $T_{\mathrm{CME}}=2 \mathrm{MK}$.

\subsection{Planetary magnetic moment and magnetosphere}

For each planet, the value of the planetary magnetic moment $\mathcal{M}$ is estimated by taking the geometrical mean of the maximum and minimum result obtained by different scaling laws. The associated uncertainty was discussed by Grießmeier et al. (2007). The different scaling laws are compared, e.g., by Farrell et al. $(1999)^{1}$, Grießmeier et al. (2004) and Grießmeier (2006). In order to be able to apply these scaling laws, some assumptions on the planetary size and structure are required. The variables required in the scaling laws are $r_{\mathrm{c}}$ (the radius of the dynamo region within the planet), $\rho_{\mathrm{c}}$ (the density within this region), $\sigma$ (the conductivity within this region) and $\omega$ (the planetary rotation rate).

The size of the planetary core $r_{\mathrm{c}}$ and its density $\rho_{\mathrm{c}}$ The density profile within the planet $\rho(r)$ is obtained by describing the planet as a polytropic gas sphere, using the solution of the LaneEmden equation (Chandrasekhar 1957; Sánchez-Lavega 2004):

$\rho(r)=\left(\frac{\pi M_{\mathrm{p}}}{4 R_{\mathrm{p}}^{3}}\right) \frac{\sin \left(\pi \frac{r}{R_{\mathrm{p}}}\right)}{\left(\pi \frac{r}{R_{\mathrm{p}}}\right)}$.

${ }^{1}$ Containing a typo in the sixth equation of the Appendix. 
The size of the planetary core $r_{\mathrm{c}}$ is found by searching for the value of $r$ where the density $\rho(r)$ becomes large enough for the transition to the liquid-metallic phase (Sánchez-Lavega 2004; Grießmeier et al. 2005; Grießmeier 2006). The transition was assumed to occur at a density of $700 \mathrm{~kg} / \mathrm{m}^{3}$, which is consistent with the range of parameters given by Sánchez-Lavega (2004). For Jupiter, we obtain $r_{\mathrm{c}}=0.85 R_{\mathrm{J}}$.

The average density in the dynamo region $\rho_{\mathrm{c}}$ is then obtained by averaging the density $\rho(r)$ over the range $0 \leq r \leq r_{\mathrm{c}}$. For Jupiter, we obtain $r_{\mathrm{c}} \approx 1800 \mathrm{~kg} \mathrm{~m}^{-3}$.

The planetary rotation rate $\omega$ Depending on the orbital distance of the planet and the timescale for synchronous rotation $\tau_{\text {sync }}$ (which is derived in Appendix B), three cases can be distinguished:

1. For planets at small enough distances for which the timescale for tidal locking is small (i.e. $\tau_{\text {sync }} \leq 100 \mathrm{Myr}$ ), the rotation period is taken to be synchronised with the orbital period $\left(\omega=\omega_{\mathrm{f}} \approx \omega_{\text {orbit }}\right)$, which is known from measurements. This case will be denoted by "TL". Typically, this results in smaller rotation rates for tidally locked planets than for freely rotating planets.

2. Planets with distances resulting in $100 \mathrm{Myr} \leq \tau_{\text {sync }} \leq$ 10 Gyr may or may not be subject to tidal locking. This will, for example, depend on the exact age of the planetary system, which is typically in the order of a few Gyr. For this reason, we calculate the expected characteristics of these "potentially locked" planets twice: once with tidal locking (denoted by "(TL)") and once without tidal locking (denoted by "(FR)").

3. For planets far away from the central star, the timescale for tidal locking is very large. For planets with $\tau_{\text {sync }} \geq 10 \mathrm{Gyr}$, the effect of tidal interaction can be neglected. In this case, the planetary rotation rate can be assumed to be equal to the initial rotation rate $\omega_{\mathrm{i}}$, which is assumed to be equal to the current rotation rate of Jupiter, i.e. $\omega=\omega_{\mathrm{J}}$ with $\omega_{\mathrm{J}}=1.77 \times$ $10^{-4} \mathrm{~s}^{-1}$. This case will be denoted by "FR".

Note that tidal interaction does not perfectly synchronise the planetary rotation to its orbit. Thermal atmospheric tides resulting from stellar heating can drive planets away from synchronous rotation (Showman \& Guillot 2002; Correia et al. 2003; Laskar \& Correia 2004). According to Showman \& Guillot (2002), the corresponding error for $\omega$ could be as large as a factor of two. On the basis of the example of $\tau$ Bootis $b$, Grießmeier et al. (2007) show that the effect of imperfect tidal locking (in combination with the spread of the results found by different scaling laws) can lead to magnetic moments and thus emission frequencies up to a factor 2.5 higher than for the nominal case. Keeping this "error bar" in mind, we will nevertheless consider only the reference case in this work.

The conductivity in the planetary core $\sigma_{\mathrm{c}}$ Finally, the conductivity in the dynamo region of extrasolar planets remains to be evaluated. According to Nellis (2000), the electrical conductivity remains constant throughout the metallic region. For this reason, it is not necessary to average over the volume of the conducting region. As the magnetic moment scaling is applied relative to Jupiter, only the relative value of the conductivity, i.e. $\sigma / \sigma_{\mathrm{J}}$ is required. In this work, the conductivity is assumed to be the same for extrasolar gas giants as for Jupiter, i.e. $\sigma / \sigma_{\mathrm{J}}=1$.

The size of the magnetosphere The size of the planetary magnetosphere $R_{\mathrm{s}}$ is calculated with the parameters determined above for the stellar wind and the planetary magnetic moment. For a given planetary orbital distance $d$, of the different pressure contributions only the magnetospheric magnetic pressure is a function of the distance to the planet. Thus, the standoff distance $R_{\mathrm{s}}$ is found from the pressure equilibrium (Grießmeier et al. 2005):

$R_{\mathrm{s}}(d)=\left[\frac{\mu_{0} f_{0}^{2} \mathcal{M}^{2}}{8 \pi^{2}\left(m n(d) v_{\mathrm{eff}}(d)^{2}+2 n(d) k_{B} T\right)}\right]^{1 / 6}$.

Here, $m$ is the proton's mass, and $f_{0}$ is the form factor of the magnetosphere. It describes the magnetic field created by the magnetopause currents. For a realistic magnetopause shape, a factor $f_{0}=1.16$ is used (Voigt 1995). In term of units normalized to Jupiter's units, this is equivalent to

$R_{\mathrm{S}}(d) \approx 40 R_{\mathrm{J}}\left[\frac{\widetilde{\mathcal{M}^{2}}}{\tilde{n}(d) \widetilde{v_{\mathrm{eff}}}(d)^{2}+\frac{2 \tilde{n}(d) k_{B} T}{m v_{\mathrm{eff}, \mathrm{J}}{ }^{2}}}\right]^{1 / 6}$,

with $\tilde{n}(d)=n(d) / n_{\mathrm{J}}, \widetilde{v_{\text {eff }}}(d)=v_{\text {eff }}(d) / v_{\text {eff }, \mathrm{J}}, n_{\mathrm{J}}=2.0 \times 10^{5} \mathrm{~m}^{-3}$, and $v_{\text {eff } \mathrm{J}}=520 \mathrm{~km} \mathrm{~s}^{-1}$.

Note that in a few cases, especially for planets with very weak magnetic moments and/or subject to dense and fast stellar winds of young stars, Eq. (30) yields standoff distances $R_{\mathrm{s}}<R_{\mathrm{p}}$, where $R_{\mathrm{p}}$ is the planetary radius. Because the magnetosphere cannot be compressed to sizes smaller than the planetary radius, we set $R_{\mathrm{s}}=R_{\mathrm{p}}$ in those cases.

\subsection{Additional parameters}

The other required parameters are obtained from the following sources:

- The stellar ages $t_{\star}$ are taken from Saffe et al. (2005, who gave ages for 112 exoplanet host stars). Saffe et al. (2005) compare stellar age estimations based on five different methods (and for one of them they use two different calibrations), some of which give more reliable results than others. Because not all of these methods are applicable to all stars, we use the age estimations in the following order or preference: chromospheric age for ages below 5.6 Gyr (using the D93 calibration), isochrone age, chromospheric age for ages above 5.6 Gyr (using the D93 calibration), metallicity age. Note that error estimates for the two most reliable methods, namely isochrone and chromospheric ages, are already relatively large (30-50\%). In those cases where the age is not known, a default value of $5.2 \mathrm{Gyr}$ is used (the median chromospheric age found by Saffe et al. 2005). This relatively high average age is due to a selection effect (planet detection by radial velocity method is easier to achieve for older, more slowly rotating stars). As the uncertainty of the radio flux estimation becomes very large for low stellar ages (Grießmeier et al. 2005), we use a minimum stellar age of $0.5 \mathrm{Gyr}$.

- For the solid angle of the beam, we assume the emission to be analogous to the dominating contributions of Jupiter's radio emission and use $\Omega=1.6 \mathrm{sr}$ (Zarka et al. 2004).

\section{Expected radio flux for know exoplanets}

\subsection{The list of known exoplanets}

Table $1^{2}$ shows what radio emission we expect from the presently known exoplanets (13.1.2007). It contains the

\footnotetext{
2 Table 1 is only available in electronic form at the CDS.
} 
maximum emission frequency according to Eq. (8), the plasma frequency in the stellar wind at the planetary location according to Eq. (9) and the expected radio flux according to the magnetic model (1), the kinetic model (2), and the kinetic CME model (4). The unipolar interaction model is discussed in the text below. Table 1 also contains values for the expected planetary mass $M_{\mathrm{p}}$, its radius $R_{\mathrm{p}}$ and its planetary magnetic dipole moment $\mathcal{M}$. For each planet, we note whether tidal locking should be expected.

Note that http://exoplanet.eu contains a few more planets than Table 1, because for some planets, essential data required for the radio flux estimation are not available (typically $s$, the distance to the observer).

The numbers given in Table 1 are not accurate results, but should be regarded as refined estimations intended to guide observations. Still, the errors and uncertainties involved in these estimations can be considerable. As was shown in Grießmeier et al. (2007), the uncertainty on the radio flux at Earth, $\Phi$, is dominated by the uncertainty in the stellar age $t_{\star}$ (for which the error is estimated as $\approx 50 \%$ by Saffe et al. 2005). For the maximum emission frequency, $f_{\mathrm{c}}^{\max }$, the error is determined by the uncertainty in the planetary magnetic moment $\mathcal{M}$, which is uncertain by a factor of two. For the planet $\tau$ Bootes b, these effects translate into an uncertainty of almost one order of magnitude for the flux (the error is smaller for planets around stars of solar age), and an uncertainty of a factor of 2-3 for the maximum emission frequency. This error estimate is derived and discussed in more detail by Grießmeier et al. (2007).

The results given in Table 1 cover the following range:

- The maximum emission frequency found to lie beween 0 to almost $200 \mathrm{MHz}$. However, all planets with $f_{\mathrm{c}}^{\max }>70 \mathrm{MHz}$ have negligible flux. Also note that any emission with $f_{\mathrm{c}} \leq 5$ to $10 \mathrm{MHz}$ will not be detectable on Earth because it cannot propagate through the Earth's ionosphere ("ionospheric cutoff"). For this reason, the most appropriate frequency window for radio observations seems to be between 10 and $70 \mathrm{MHz}$.

- The radio flux according to the magnetic energy model, $\Phi_{\text {sw,mag }}$ lies between 0 and 5 Jy (for GJ 436 b). For 15 candidates, $\Phi_{\mathrm{sw} \text {,mag }}$ is larger than $100 \mathrm{mJy}$, and for 37 candidates it is above $10 \mathrm{mJy}$.

- The flux prediction according to the kinetic energy model, $\Phi_{\text {sw,kin }}$ is much lower than the flux according to the magnetic energy model. Only in one case it exceeds the value of $10 \mathrm{mJy}$.

- The increased stellar wind density and velocity during a $C M E$ leads to a strong increase of the radio flux when compared to the kinetic energy model. Correspondingly, $\Phi_{\mathrm{CME}, \mathrm{kin}}$ exceeds $100 \mathrm{mJy}$ in 3 cases and $10 \mathrm{mJy}$ in 11 cases.

- Table 1 does not contain flux estimations for the unipolar interaction model. The reason is that the condition given by Eq. (11) is not satisfied in any of the studied cases. This is consistent with the result of Zarka $(2006,2007)$, who found that stars 100 times as strongly magnetised as the sun are required for this type of emission. The approach taken in Sect. 3.2 for the estimation of stellar magnetic fields does not yield such strong magnetic fields for stars with ages $>0.5$ Gyr. Stronger magnetic fields are possible (e.g. for younger stars). Strongly magnetised stars (even those without known planets) could be defined as specific targets to test this model.

- The plasma frequency in the stellar wind, $f_{\mathrm{p}, \mathrm{sw}}$, is negligibly small in most cases. For a few planets, however, it is of the same order of magnitude as the maximum emission frequency. In these cases, the condition given by Eq. (10) makes the escape of the radio emission from its source towards the observer impossible. Of the 197 planets of the current census, Eq. (10) is violated in 8 cases. If one takes into account the uncertainty of the stellar age (30-50\%, see Saffe et al. 2005), an uncertainty of similar size is introduced for the plasma frequency: in the example of $\tau$ Bootis, the age uncertainty translates into a variation of up to $50 \%$ for the plasma frequency. With such error bars, between 6 and 14 planets are affected by Eq. (10). None of the best targets are affected.

- The expected planetary magnetic dipole moments lie between 0 and 5.5 times the magnetic moment of Jupiter. However, the highest values are found only for very massive planets: planets with masses $M \leq 2 M_{\mathrm{J}}$ have magnetic moments $\mathcal{M} \leq 2 \mathcal{M}_{\mathrm{J}}$. For planets with masses of $M \leq M_{\mathrm{J}}$, the models predict magnetic moments $\mathcal{M} \leq 1.1 \mathcal{M}_{\mathrm{J}}$.

The results of Table 1 confirm that the different models for planetary radio emission lead to very different results. The largest fluxes are found for the magnetic energy model, followed by the CME model and the kinetic energy model. This is consistent with previous expectations (Zarka et al. 2001b; Zarka 2006; Grießmeier et al. 2006b; Zarka 2007; Grießmeier et al. 2007). The unipolar interaction model does not lead to observable emission for the presently known exoplanets. Furthermore, the impact of tidal locking is clearly visible in the results. As it is currently not clear which of these models best describes the auroral radio emission, it is not sufficient to restrict oneself to one scaling law (e.g. the one yielding the largest radio flux). For this reason, all possible models have to be considered. Once exoplanetary radio emission is detected, observations will be used to constrain and improve the models.

Table 1 also shows that planets subject to tidal locking have a smaller magnetic moment and thus a lower maximum emission frequency than freely rotating planets. The reduced bandwidth of the emission can lead to an increase of the radio flux, but frequently emission is limited to frequencies not observable on earth (i.e. below the ionospheric cutoff).

The results of Table 1 are visualized in Fig. 1 (for the magnetic energy model), Fig. 2 (for the $C M E$ model) and Fig. 3 (for the kinetic energy model). The predicted planetary radio emission is denoted by open triangles (two for each "potentially locked" planet, otherwise one per planet). The typical uncertainties (approx. one order of magnitude for the flux, and a factor of 2-3 for the maximum emission frequency) are indicated by the arrows in the upper right corner. The sensitivity limit of previous observation attempts are shown as filled symbols and as solid lines (a more detailed comparison of these observations can be found in Zarka 2004; Grießmeier et al. 2006a; Grießmeier 2006). The expected sensitivity of new and future detectors (for $1 \mathrm{~h}$ integration and $4 \mathrm{MHz}$ bandwidth, or any equivalent combination) is shown for comparison. Dashed line: upgraded UTR-2, dash-dotted lines: low band and high band of LOFAR, left dotted line: LWA, right dotted line: SKA. The instruments' sensitivities are defined by the radio sky background. For a given instrument, a planet is observable if it is located either above the instrument's symbol or above and to its right. Again, large differences in expected flux densities are apparent between the different models. On average, the magnetic energy model yields the largest flux densities, and the kinetic energy model yields the lowest values. Depending on the model, between one and three planets are likely to be observable using the upgraded system of UTR-2. Somewhat higher numbers 


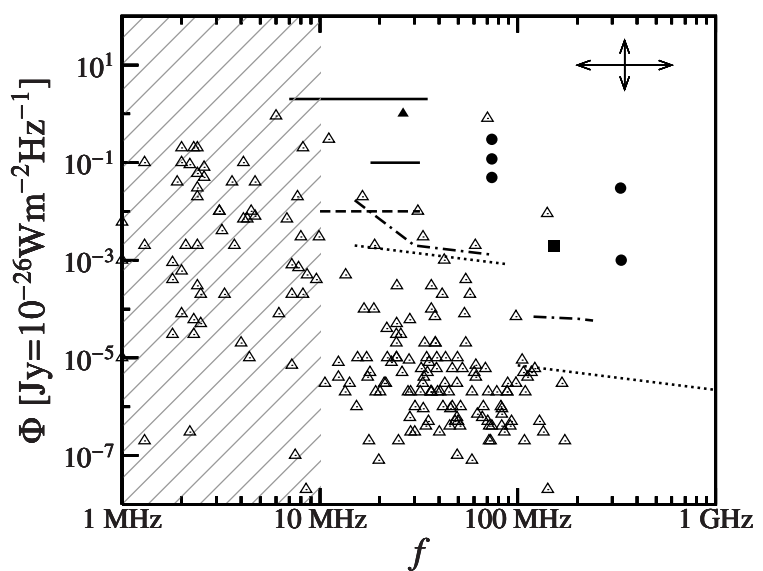

Fig. 1. Maximum emission frequency and expected radio flux for known extrasolar planets according to the magnetic energy model, compared to the limits of past and planned observation attempts. Open triangles: Predictions for planets. Solid lines and filled circles: previous observation attempts at the UTR-2 (solid lines), at Clark Lake (filled triangle), at the VLA (filled circles), and at the GMRT (filled rectangle). For comparison, the expected sensitivity of new detectors is shown: upgraded UTR-2 (dashed line), LOFAR (dash-dotted lines, one for the low band and one for the high band antenna), LWA (left dotted line) and SKA (right dotted line). Frequencies below $10 \mathrm{MHz}$ are not observable from the ground (ionospheric cutoff). Typical uncertainties are indicated by the arrows in the upper right corner.

are found for LOFAR. Considering the uncertainties mentioned above, these numbers should not be taken literally, but should be seen as an indicator that while observation seem feasible, the number of suitable candidates is rather low. It can be seen that the maximum emission frequency of many planets lies below the ionospheric cutoff frequency, making earth-based observation of these planets impossible. A moon-based radio telescope however would give access to radio emission with frequencies of a few MHz (Zarka 2007). As can be seen in Figs. 1-3, this frequency range includes a significant number of potential target planets with relatively high flux densities.

Figures 1-3 also show that the relatively high frequencies of the LOFAR high band and of the SKA telescope are probably not very well suited for the search for exoplanetary radio emission. These instruments could, however, be used to search for radio emission generated by unipolar interaction between planets and strongly magnetised stars.

\subsection{A few selected cases}

According to our analysis, the best candidates are:

- HD $41004 \mathrm{~B}$ b, which is the best case in the magnetic energy model with emission above $1 \mathrm{MHz}$. Note that the mass of this object is higher than the upper limit for planets $\left(\approx 13 M_{\mathrm{J}}\right)$, so that it probably is a brown dwarf and not a planet.

- Epsilon Eridani b, which is the best case in the kinetic energy model.

- Tau Boo b, which is the best case in the magnetic energy model with emission above the ionospheric cutoff $(10 \mathrm{MHz})$.

- HD 189733 b, which is the best case in both the magnetic energy model and in the $C M E$ model which has emission above $1 \mathrm{MHz}$.

- Gliese $876 \mathrm{c}$, which is the best case in the $C M E$ model with emission above the ionospheric cutoff (10 MHz).

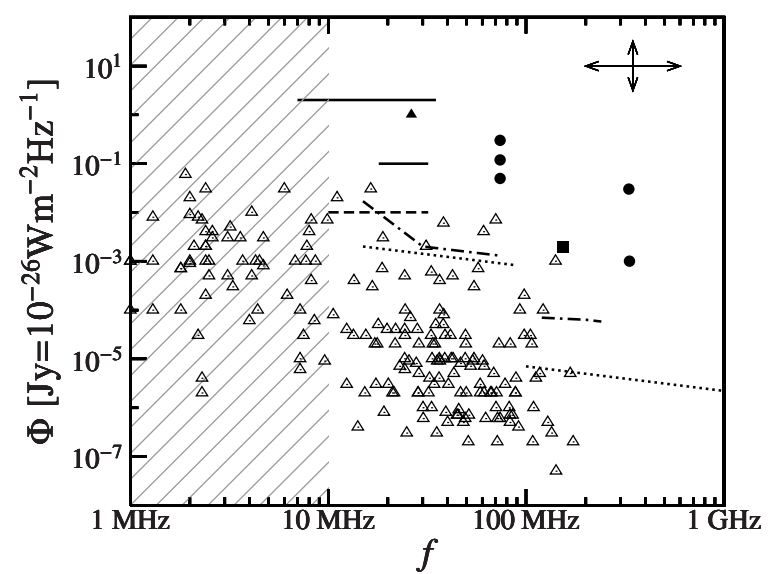

Fig. 2. Maximum emission frequency and expected radio flux for known extrasolar planets according to the $C M E$ model, compared to the limits of past and planned observation attempts. Open triangles: predictions for planets. All other lines and symbols are as defined in Fig. 1.

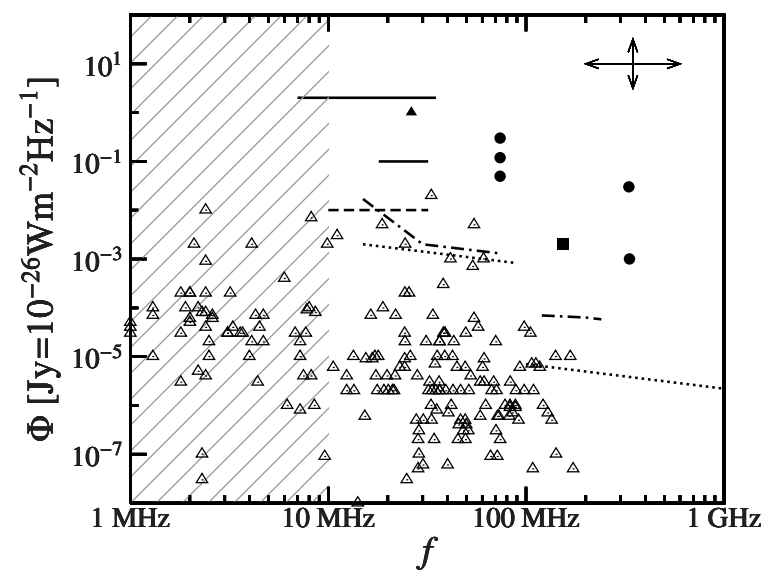

Fig. 3. Maximum emission frequency and expected radio flux for known extrasolar planets according to the kinetic energy model, compared to the limits of past and planned observation attempts. Open triangles: predictions for planets. All other lines and symbols are as defined in Fig. 1.

- HD 73256 b, which has emission above 100 mJy in the magnetic energy model and which is the second best planet in the kinetic energy model.

- GJ 3021 b, which is the third best planet in the kinetic energy model.

To this list, one should add the planets around Ups And (b, c and d) and HD 179949 b, whose parent stars exhibit an increase of the chromospheric emission of about 1-2\% (Shkolnik et al. 2003, 2004, 2005). The observations indicate one maximum per planetary orbit, a "Hot Spot" in the stellar chromosphere which is in phase with the planetary orbit. The lead angles observed by Shkolnik et al. (2003) and Shkolnik et al. (2005) were recently explained with an Alfvén-wing model using realistic stellar wind parameters obtained from the stellar wind model by Weber and Davis (Preusse 2006; Preusse et al. 2006). This indicates that a magnetised planet is not required to describe the present data. The presence of a planetary magnetic field could, however, be proven by the existence of planetary radio emission. Although our model does not predict high radio fluxes from these planets (see Table 1), the high chromospheric flux shows that a strong interaction is taking place. As a possible solution of this problem, an intense stellar magnetic field was suggested (Zarka 2006, 2007). In that case, Table 1 underestimates the radio emission of 
Ups And b, c, d and HD 179949 b, making these planets interesting candidates for radio observations (e.g. through the magnetic energy model or the unipolar interaction model). For this reason, it would be especially interesting to obtain measurements of the stellar magnetic field for these two planet-hosting stars (e.g. by the method of Catala et al. 2007).

Considering the uncertainties mentioned above, it is important not to limit observations attempts to these best cases. The estimated radio characteristics should only be used as a guide (e.g. for the target selection, or for statistical analysis), but individual results should not be regarded as precise values.

\subsection{Statistical discussion}

It may seem surprising that so few good candidates are found among the 197 examined exoplanets. However, when one checks the list of criteria for "good" candidates (e.g. Grießmeier et al. 2006a), it is easily seen that only a few good targets can be expected: (a) the planet should be close to the Earth (otherwise the received flux is too weak). About $70 \%$ of the known exoplanets are located within $50 \mathrm{pc}$, so that this is not a strong restriction; (b) a strongly magnetised system is required (especially to obtain frequencies above the ionospheric cutoff). For this reason, the planet should be massive (as seen above, we find magnetic moments $\mathcal{M} \geq 2 \mathcal{M}_{\mathrm{J}}$ only for planets with masses $M \geq 2 M_{\mathrm{J}}$ ). About $60 \%$ of the known exoplanets are at least as massive as Jupiter (but only $40 \%$ have $M_{\mathrm{p}} \geq 2.0 M_{\mathrm{J}}$ ); (c) the planet should be located close to its host star to allow for strong interaction (dense stellar wind, strong stellar magnetic field). Only $25 \%$ of the known exoplanets are located within $0.1 \mathrm{AU}$ of their host star.

By multiplying these probabilities, one finds that close ( $s \leq$ $50 \mathrm{pc})$, heavy $\left(M_{\mathrm{p}} \geq 2.0 M_{\mathrm{J}}\right)$, close-in $(d \leq 0.1 \mathrm{AU})$ planets would represent $8 \%(\approx 15$ of 197 planets $)$ of the current total if the probabilities for the three conditions were independent. However, this is not the case. In the current census of exoplanets, a correlation between planetary mass and orbital distance is clearly evident, with a lack of close-in massive planets (see e.g. Udry et al. 2003). This is not a selection effect, as massive close-in planets should be easier to detect than low-mass planets. This correlation was explained by the stronger tidal interaction effects for massive planets, leading to a faster decrease of the planetary orbital radius until the planet reaches the stellar Roche limit and is effectively destroyed (Pätzold \& Rauer 2002; Jiang et al. 2003). Because of this mass-orbit correlation, the fraction of good candidates is somewhat lower (approx. 2\%, namely 3 of 197 planets: HD 41004 B b, Tau Boo b, and HD 162020 b).

\subsection{Comparison to previous results}

A first comparative study of expected exoplanetary radio emission from a large number of planets was performed by Lazio et al. (2004), who compared expected radio fluxes of 118 planets (i.e. those known as of 2003, July 1). Their results differ considerably from those given in Table 1 :

- As far as the maximum emission frequency $f_{\mathrm{c}}^{\max }$ is concerned, our results are considerably lower than the frequencies given by Lazio et al. (2004). For Tau Bootes, their maximum emission frequency is six times larger than our result. For planets heavier than Tau Bootes, the discrepancy is even larger, reaching more than one order of magnitude for the very heavy cases (e.g. HD 168433c, for which they predict radio emission with frequencies up to $2670 \mathrm{MHz}$ ).
These differences have several reasons: firstly, Farrell et al. (1999) and Lazio et al. (2004) assume that $R_{\mathrm{p}}=R_{\mathrm{J}}$. Also, these works rely on the magnetic moment scaling law of Blackett (1947), which has a large exponent in $r_{\mathrm{c}}$. This scaling law should not be used, as it was experimentally disproven (Blackett 1952). Thirdly, these works make uses $r_{\mathrm{c}} \propto M_{\mathrm{p}}^{1 / 3}$. Especially for planets with large masses like $\tau$ Bootes, this yields unrealistically large core radii (even $r_{\mathrm{c}}>R_{\mathrm{p}}$ in some cases), magnetic moments, and emission frequencies. Note that a good estimation of the emission frequency is particularly important, because a difference of a factor of a few can make the difference between radiosignals above and below the Earth's ionospheric cutoff frequency.

- The anticipated radio flux obtained with the kinetic energy model $\Phi_{\text {sw,kin }}$ is much lower than the estimates of Lazio et al. (2004). Typically, the difference is approximately two orders of magnitude, but this varies strongly from case to case. For example, for Tau Boo b, our result is smaller by a factor of 30 (where the difference is partially compensated by the low stellar age which increases our estimation), for Ups And b, the results differ by a factor of 220 , and for Gliese $876 \mathrm{c}$, the difference is as large as a factor 6300 (which is partially due to the fact we take into account the small stellar radius and the high stellar age).

- As was mentioned above, the analysis of the jovian radio emission allows to define three terms for the typical radio spectrum: (a) the power during average conditions; (b) the average power during periods of high activity; and (c) the peak power (Zarka et al. 2004). When comparing the results of our Table 1 to those of Lazio et al. (2004), one has to note that their Table I gives the peak power, while the results in our Table 1 were obtained using the average power during periods of high activity. Similarly to Farrell et al. (1999), Lazio et al. (2004) assume that the peak power caused by variations of the stellar wind velocity is two orders of magnitude higher than the average power. However, the values Farrell et al. (1999) use for average conditions correspond to periods of high activity, which are less than one order of magnitude below the peak power (Zarka et al. 2004). During periods of peak emission, the value given in our Table 1 would be increased by the same amount (approximately a factor of 5, see Zarka et al. 2004). For this reason, the peak radio flux is considerably overestimated in these studies.

- Estimated radio fluxes according to the magnetic energy model and the $C M E$ model have not yet been published for large numbers of planets. This is the first time the results from these models are compared for a large number of planets.

\section{Conclusions}

Predictions concerning the radio emission from all presently known extrasolar planets were presented. The main parameters related to such an emission were analyzed, namely the planetary magnetic dipole moments, the maximum frequency of the radio emission, the radio flux densities, and the possible escape of the radiation towards a remote observer.

We compared the results obtained with various theoretical models. Our results confirm that the four different models for planetary radio emission lead to very different results. As expected, the largest fluxes are found for the magnetic energy model, followed by the $C M E$ model and the kinetic energy model. The results obtained by the latter model are found to be less optimistic than by previous studies. The unipolar interaction model does not lead to observable emission for any of the 
currently known planets. As it is currently not clear which of these models best describes the auroral radio emission, it is not sufficient to restrict oneself to one scaling law (e.g. the one yielding the largest radio flux). Once exoplanetary radio emission is detected, observations will be used to constrain and improve the model.

These results will be particularly useful for the target selection of current and future radio observation campaigns (e.g. with the VLA, GMRT, UTR-2 and with LOFAR). We have shown that observation seem feasible, but that the number of suitable candidates is relatively low. The best candidates appear to be HD 41004 B b, Epsilon Eridani b, Tau Boo b, HD 189733 b, Gliese $876 \mathrm{c}$, HD $73256 \mathrm{~b}$, and GJ $3021 \mathrm{~b}$. The observation of some of these candidates is in progress.

Acknowledgements. We thank J. Schneider for providing data via "The extrasolar planet encyclopedia" (http://exoplanet. eu/), I. Baraffe and C. Vocks for helpful discussions concerning planetary radii. We would also like to thank the anonymous referee for his helpful comments. This study was jointly performed within the ANR project "La détection directe des exoplanètes en ondes radio" and within the LOFAR transients key project (TKP). J.-M. G. was supported by the french national research agency (ANR) within the project with the contract number NT05-1_42530 and partially by Europlanet (N3 activity). P.Z. acknowledges support from the International Space Science Institute (ISSI) within the ISSI team "Search for Radio Emissions from Extra-Solar Planets".

\section{References}

Baraffe, I., Chabrier, G., Barman, T. S., Allard, F., \& Hauschildt, P. H. 2003, A\&A, 402, 701

Baraffe, I., Chabrier, G., Barman, T. S., et al. 2005, A\&A, 436, L47

Blackett, P. M. S. 1947, Nature, 159, 658

Blackett, P. M. S. 1952, Phil. Trans. R. Soc. A, 245, 309

Bodenheimer, P., Laughlin, G., \& Lin, D. N. C. 2003, ApJ, 592, 555

Burrows, A., Guillot, T., Hubbard, W. B., et al. 2000, ApJ, 534, L97

Burrows, A., Sudarsky, D., \& Hubbard, W. B. 2003, ApJ, 594, 545

Burrows, A., Hubeny, I., Hubbard, W. B., Sudarsky, D., \& Fortney, I. J. 2004, ApJ, 610, L53

Cain, J. C., Beaumont, P., Holter, W., Wang, Z., \& Nevanlinna, H. 1995, J. Geophys. Res., 100, 9439

Catala, C., Donati, J.-F., Shkolnik, E., Bohlender, D., \& Alecian, E. 2007, MNRAS, 374, L42

Chandrasekhar, S. 1957, An Introduction to the Study of Stellar Structure, Dover Books on Astronomy and Astrophysics (New York: Dover Publications, Inc.) Collier Cameron, A., \& Jianke, L. 1994, MNRAS, 269, 1099

Correia, A. C. M., Laskar, J., \& de Surgy, O. N. 2003, Icarus, 163, 1

Desch, M. D., \& Kaiser, M. L. 1984, Nature, 310, 755

Dobbs-Dixon, I., Lin, D. N. C., \& Mardling, R. A. 2004, ApJ, 610, 464

Farrell, W. M., Desch, M. D., \& Zarka, P. 1999, J. Geophys. Res., 104, 14025

Farrell, W. M., Lazio, T. J. W., Zarka, P., et al. 2004, Planet. Space Sci., 52, 1469

Goldreich, P., \& Soter, S. 1966, Icarus, 5, 375

Grießmeier, J.-M. 2006, Ph.D. Thesis, Technische Universität Braunschweig, ISBN 3-936586-49-7, Copernicus-GmbH Katlenburg-Lindau,

URL: http: //www . digibib. tu-bs . de/?docid=00013336

Grießmeier, J.-M., Stadelmann, A., Penz, T., et al. 2004, A\&A, 425, 753

Grießmeier, J.-M., Motschmann, U., Mann, G., \& Rucker, H. O. 2005, A\&A, 437, 717

Grießmeier, J.-M., Motschmann, U., Glassmeier, K.-H., Mann, G., \& Rucker, H. O. 2006a, in Tenth Anniversary of 51 Peg-b: Status of and Prospects for hot Jupiter studies, ed. L. Arnold, F. Bouchy, \& C. Moutou (Platypus Press), 259, URL:

http://www .obs-hp.fr/www/pubs/Coll51Peg/proceedings.html

Grießmeier, J.-M., Motschmann, U., Khodachenko, M., \& Rucker, H. O. 2006b, in Planetary Radio Emissions VI, ed. H. O. Rucker, W. S. Kurth, \& G. Mann (Vienna: Austrian Academy of Sciences Press), 571

Grießmeier, J.-M., Preusse, S., Khodachenko, M., et al. 2007, Planet. Space Sci., 55,618

Gu, P.-G., Lin, D. N. C., \& Bodenheimer, P. H. 2003, ApJ, 588, 509

Guillot, T., Santos, N. C., Pont, F., et al. 2006, A\&A, 453, L21

Halbwachs, J. L., Mayor, M., \& Udry, S. 2005, A\&A, 431, 1129

Hilgers, A. 1992, Geophys. Res. Lett., 19, 237

Holzwarth, V., \& Jardine, M. 2007, A\&A, 463, 11

Hubbard, W. B. 1984, Planetary interiors (New York: Van Nostrand Reinhold Co.)
Jiang, I.-G., Ip, W.-H., \& Yeh, L.-C. 2003, ApJ, 582, 449

Khodachenko, M. L., Lammer, H., Lichtenegger, H. I. M., et al. 2007a, Planet. Space Sci., 55, 631

Khodachenko, M. L., Ribas, I., Lammer, H., et al. 2007b, Astrobiology, 7, 167

Laskar, J., \& Correia, A. C. M. 2004, in Extrasolar Planets: Today and Tomorrow, ed. J.-P. Beaulieu, A. Lecavelier des Etangs, \& C. Terquem, ASP Conf. Ser., 321, 401

Lazio, T. J. W., Farrell, W. M., Dietrick, J., et al. 2004, ApJ, 612, 511

Le Quéau, D., Pellat, R., \& Roux, A. 1985, Ann. Geophys., 3, 273

Levrard, B., Correia, A. C. M., Chabrier, G., et al. 2007, A\&A, 462, L5

Lynden-Bell, D., \& O’Dwyer, J. P. 2001 [arXiv: astro-ph/0104450]

Lynden-Bell, D., \& Tout, C. A. 2001, ApJ, 558, 1

MacDonald, G. J. F. 1964, Rev. Geophys., 2, 467

Majid, W., Winterhalter, D., Chandra, I., et al. 2006, in Planetary Radio Emissions VI, ed. H. O. Rucker, W. S. Kurth, \& G. Mann (Vienna: Austrian Academy of Sciences Press), 589

Mann, G., Jansen, F., MacDowall, R. J., Kaiser, M. L., \& Stone, R. G. 1999, A\&A, 348, 614

Marcy, G. W., Butler, R. P., Williams, E., et al. 1997, ApJ, 481, 926

Mariani, F., \& Neubauer, F. M. 1990, in Physics of the Inner Heliosphere, ed. R. Schwenn, \& E. Marsch (Berlin: Springer-Verlag), 1, 183

Murray, C. D., \& Dermott, S. F. 1999, Solar System Dynamics (Cambridge: Cambridge University Press)

Nellis, W. J. 2000, Planet. Space Sci., 48, 671

Newkirk, Jr., G. 1980, in The Ancient Sun: Fossil Record in the Earth, Moon and Meteorites, ed. R. O. Pepin, J. A. Eddy, \& R. B. Merrill, 293

Parker, E. N. 1958, ApJ, 128, 664

Pätzold, M., \& Rauer, H. 2002, ApJ, 568, L117

Peale, S. J. 1999, ARA\&A, 37, 533

Preusse, S. 2006, Ph.D. Thesis, Technische Universität Braunschweig, Copernicus-GmbH Katlenburg-Lindau

Preusse, S., Kopp, A., Büchner, J., \& Motschmann, U. 2005, A\&A, 434, 1191 Preusse, S., Kopp, A., Büchner, J., \& Motschmann, U. 2006, A\&A, 460, 317

Prölss, G. W. 2004, Physics of the Earth's Space Environment (Berlin: SpringerVerlag)

Ryabov, V. B., Zarka, P., \& Ryabov, B. P. 2004, Planet. Space Sci., 52, 1479

Saffe, C., Gómez, M., \& Chavero, C. 2005, A\&A, 443, 609

Sánchez-Lavega, A. 2004, ApJ, 609, L87

Sato, B., Fischer, D. A., Henry, G. W., et al. 2005, ApJ, 633, 465

Shkolnik, E., Walker, G. A. H., \& Bohlender, D. A. 2003, ApJ, 597, 1092

Shkolnik, E., Walker, G. A. H., \& Bohlender, D. A. 2004, ApJ, 609, 1197

Shkolnik, E., Walker, G. A. H., Bohlender, D. A., Gu, P.-G., \& Kürster, M. 2005, ApJ, 622, 1075

Showman, A. P., \& Guillot, T. 2002, A\&A, 385, 166

Stevens, I. R. 2005, MNRAS, 356, 1053

Tout, C. A., Pols, O. R., Eggleton, P. P., \& Han, Z. 1996, MNRAS, 281, 257

Treumann, R. A. 2000, in Geophysical monograph series, Vol. 119, Radio Astronomy at Long Wavelengths, ed. R. Stone, K. W. Weiler, M. L. Goldstein, \& J.-L. Bougeret, 13

Udry, S., Mayor, M., \& Santos, N. C. 2003, A\&A, 407, 369

Voigt, G.-H. 1995, in Handbook of atmospheric electrodynamics, ed. H. Volland, Vol. II (CRC Press), 333

Weber, E. J., \& Davis, Jr., L. 1967, ApJ, 148, 217

Winterhalter, D., Kuiper, T., Majid, W., et al. 2006, in Planetary Radio Emissions VI, ed. H. O. Rucker, W. S. Kurth, \& G. Mann (Austrian Academy of Sciences Press, Vienna), 595

Wood, B. E. 2004, Living Rev. Solar Phys., 1, 2, URL:

http://www . livingreviews . org/lrsp-2004-2, accessed on 23 February 2007

Wood, B. E., Müller, H.-R., Zank, G. P., \& Linsky, J. L. 2002, ApJ, 574, 412

Wood, B. E., Müller, H.-R., Zank, G. P., Linsky, J. L., \& Redfield, S. 2005, ApJ, 628, L143

Yantis, W. F., Sullivan, III., W. T., \& Erickson, W. C. 1977, BAAS, 9, 453

Zarka, P. 2004, in Extrasolar planets: Today and Tomorrow, ed. J.-P. Beaulieu, A. Lecavelier des Etangs, \& C. Terquem, ASP Conf. Ser., 321, 160

Zarka, P. 2006, in Planetary Radio Emissions VI, ed. H. O. Rucker, W. S. Kurth, \& G. Mann (Vienna: Austrian Academy of Sciences Press), 543

Zarka, P. 2007, Planet. Space Sci., 55, 598

Zarka, P., Queinnec, J., Ryabov, B. P., et al. 1997, in Planetary Radio Emissions IV, ed. H. O. Rucker, S. J. Bauer, \& A. Lecacheux (Vienna: Austrian Academy of Sciences Press), 101

Zarka, P., Queinnec, J., \& Crary, F. J. 2001a, Planet. Space Sci., 49, 1137

Zarka, P., Treumann, R. A., Ryabov, B. P., \& Ryabov, V. B. 2001b, Astrophys. Space Sci., 277, 293

Zarka, P., Cecconi, B., \& Kurth, W. S. 2004, J. Geophys. Res., 109, A09S15 


\section{Online Material}




\section{Appendix A: An empirical mass-radius relation}

For the selection of targets for the search for radio emission from extrasolar planets, an estimation of the expected radio flux $\Phi$ and of the maximum emission frequency $f_{\mathrm{c}}^{\max }$ is required. For the calculation of these values, both the planetary mass and the planetary radius are required (see, e.g. Farrell et al. 1999; Grießmeier et al. 2007; Zarka 2007). However, only for a few planets (i.e. the 16 presently known transiting planets) both mass and radius are known.

In the absence of observational data, it is in principle possible to obtain planetary radii from numerical simulation, e.g. similar to those of Bodenheimer et al. (2003) or Baraffe et al. (2003, $2005)$, requiring one numerical run per planet. We chose instead to derive a simplified analytical fit to such numerical results.

\section{A.1. The accuracy of the fit}

The description presented here is necessarily only preliminary, as (a) numerical models are steadily further developed and improved, and as (b) more transit observations (e.g. by the COROT satellite, which was launched recently) will provide a much better database in the future. This will considerably improve our understanding of the dependence of the planetary radius on various parameters as, e.g. planetary mass, orbital distance, or stellar metallicity (as suggested by Guillot et al. 2006).

\section{A.1.1. What accuracy can we accept?}

Within the frame of the models presented in Sect. 2, an increase in $R_{\mathrm{p}}$ by $40 \%$ increases the expected radio flux by a factor of 2 , and the estimated maximum emission frequency decreases by $40 \%$. More generally, for a fixed planetary mass, $\Phi$ is roughly proportional to $R_{\mathrm{p}}^{7 / 3}$ and $f_{\mathrm{c}}^{\max }$ is approximately proportional to $R_{\mathrm{p}}^{-1}$. Thus, it appears that the assumption of a single standard radius for all planets leads to a relatively large error. Comparing this to the other uncertainties involved in the estimation of radio characteristics (these are discussed in Grießmeier et al. 2007), it seems sufficient to estimate $R_{\mathrm{p}}$ with $20 \%$ accuracy.

\section{A.1.2. What accuracy can we expect?}

Several effects limit the precision in planetary radius we can hope to achieve:

- the definition of the radius: the "transit radius" measured for transiting planets is not exactly identical to the standard 1 bar radius. The differences are of the order of about $5-10 \%$, but depend on the mass of the planet (Burrows et al. 2003, 2004). Because we compare modelled radii and observed radii without correcting for this effect, this limits the maximum precision we can potentially obtain.

- The abundance of heavy elements: The transiting planet around HD 149026 is substantially enriched in heavy elements (Sato et al. 2005). Models (Bodenheimer et al. 2003) yield a smaller radius for planets with a heavy core than for coreless planets of the same mass (more than $10 \%$ difference for small planets). For a planet with unknown radius, a strong enrichment in heavy elements cannot be ruled out, as this case cannot be distinguished observationally from a pure hydrogen giant (i.e. one without heavy elements).

For these reasons, we conclude that an analytical description which agrees with the (numerical) data within $\sim 20 \%$ seems suf- ficient. For such a description, the error introduced by the fit will not be the dominant one. To get a better result, it is not sufficient to improve the approximation for the radius estimation, but the more fundamental problems mentioned above have to be addressed.

\section{A.2. An analytical mass-radius relation}

\section{A.2.1. The influence of the planetary mass}

A simple mass-radius relation, valid within a vast mass range, has been proposed by Lynden-Bell \& O’Dwyer (2001) and Lynden-Bell \& Tout (2001):

$$
R_{\mathrm{p}}=\frac{1}{\left(\frac{4}{3} \pi \rho_{0}\right)^{1 / 3}} \frac{M_{\mathrm{p}}^{1 / 3}}{1+\left(\frac{M_{\mathrm{p}}}{M_{\max }}\right)^{2 / 3}} .
$$

The density $\rho_{0}$ is depends on the planetary atomic composition. Equation (A.1) has a maximum in $R_{\mathrm{p}}$ when $M_{\mathrm{p}}=M_{\max }$ (corresponding to the planet of maximum radius). Fitting Jupiter, Saturn and the planet of maximum radius $\left(R_{\max }=1.16 R_{\mathrm{J}}\right.$, see Hubbard 1984), we obtain $M_{\max }=3.16 M_{\mathrm{J}}$ and $\rho_{0}=$ $394 \mathrm{~kg} \mathrm{~m}^{-3}$ for a Jupiter-like mixture of hydrogen and helium ( $75 \%$ and $25 \%$ by mass, respectively). For a pure hydrogen planet, $\rho_{0}=345 \mathrm{~kg} \mathrm{~m}^{-3}$. With there parameters, Eq. (A.1) fits well the results for cold planets of Bodenheimer et al. (2003). In this work, this estimation is used for the radii of non-irradiated (cold) planets.

\section{A.2.2. The influence of the planetary age}

It is known that the radius of a planet with a given mass depends on its age. According to models for the radii of isolated planets (Baraffe et al. 2003), the assumption of a time-independent planetary radius leads to an error $\leq 11 \%$ for planets with ages above $0.5 \mathrm{Gyr}$ and with masses above $0.5 \mathrm{M}_{\mathrm{J}}$. In view of the uncertainties discussed above, this error seems acceptable for a first approximation, and we use

$R_{\mathrm{p}}\left(M_{\mathrm{p}}, t\right) \approx R_{\mathrm{p}}\left(M_{\mathrm{p}}\right)$

\section{A.2.3. The influence of the planetary orbital distance}

It is commonly expected that planets subjected to strong stellar radiation have a larger planetary radius than isolated, but otherwise identical planets. This situation is typical for "Hot Jupiters", where strong stellar irradiation is supposed to delay the planetary contraction (Burrows et al. 2000, 2003, 2004). Clearly, this effect depends on the planetary distance to its star, $d$, and on the stellar luminosity $L_{\star}$. In the following, we denote the radius increase by $r$, which we define as

$$
r=\frac{R_{\mathrm{p}}\left(M_{\mathrm{p}}, d\right)}{R_{\mathrm{p}}\left(M_{\mathrm{p}}, d=\infty\right)}
$$

Herein, $R_{\mathrm{p}}\left(M_{\mathrm{p}}, t, d\right)$ denotes the radius of a planet under the irradiation by its host star, and $R_{\mathrm{p}}\left(M_{\mathrm{p}}, t, d=\infty\right)$ is the radius of a non-irraditated, but otherwise identical planet.

Different exoplanets have vastly different host stars. A difference of a factor of two in stellar mass can result in a difference of more than order of magnitude in stellar luminosity. For this reason, it is not sufficient to take the orbital distance as the only parameter determining the radius increase by irradiation. 
Here, we select the equilibrium temperature of the planetary surface as the basic parameter. It is defined as (Bodenheimer et al. 2003)

$T_{\mathrm{eq}}=\left[\frac{(1-A) L_{\star}}{16 \pi \sigma_{\mathrm{SB}} d^{2}\left(1+e^{2} / 2\right)^{2}}\right]^{1 / 4}$,

where the planetary albedo is set as $A=0.4$ in the following. The stellar luminosity $L_{\star}$ is calculated from the stellar mass according to the analytical fit given by Tout et al. (1996) for the zero-age main sequence. In this, we assumed solar metallicity for all stars. Finally, $\sigma_{\mathrm{SB}}$ denotes the Stefan-Boltzmann constant.

Using the data of Bodenheimer et al. (2003), we use the following fit for the radius increase $r$ :

$r=1+0.05\left(\frac{T_{\mathrm{eq}}}{T_{0}}\right)^{\gamma}$.

This form was selected because it has the correct qualitative behaviour: it yields a monotonous decrease of $r$ with decreasing equilibrium temperature $T_{\mathrm{eq}}$ (increasing orbital distance $d$ ). In the limit $T_{\text {eq }} \rightarrow 0(d \rightarrow \infty)$ we find $r \rightarrow 1$. As an alternative to a continuous fit like Eq. (A.5), Lazio et al. (2004) use a stepfunction, i.e. assume an increased radius of $r=1.25$ for planets closer than a certain distance $d_{1}=0.1 \mathrm{AU}$ only. However, this treatment does not reduce the number of fit-constants. Also, for planets with orbital distances close to $d_{1}$, the results obtained with a step-function strongly depend on the somehow arbitrary choice of $d_{1}$. A continuous transition from "irradiated" to "isolated" planets is less prone to this effect.

The numerical results of Bodenheimer et al. (2003) show that the ratio $r$ also depends on the mass of the planet: for small planets, $r=1.4$ for an equilibrium temperature of $2000 \mathrm{~K}$, whereas for large planets, $r \leq 1.10$. For this reason, we allow to coefficients $T_{0}$ and $\gamma$ to vary with $M_{\mathrm{p}}$ :

$T_{0}=c_{\mathrm{t}, 1} \cdot\left(\frac{M_{\mathrm{p}}}{M_{\mathrm{J}}}\right)^{c_{\mathrm{t}, 2}}$

and

$\gamma=1.15+0.05 \cdot\left(\frac{c_{\gamma, 1}}{M_{\mathrm{p}}}\right)^{c_{\gamma, 2}}$.

With the set of coefficient $c_{\mathrm{t}, 1}=764 \mathrm{~K}, c_{\mathrm{t}, 2}=0.28, c_{\gamma, 1}=$ $0.59 M_{\mathrm{J}}$ and $c_{\gamma, 2}=1.03$, we obtain an analytical fit to the numerical results of Bodenheimer et al. (2003). The maximum deviation from the numerical results is below $10 \%$ (cf. Fig. A.1).

For comparison, Fig. A.1 also shows the value of $r$ for the transiting exoplanets OGLE-TR-10b, OGLE-TR-56b, OGLE-TR-111b, OGLE-TR-113b, OGLE-TR-132b, XO-1b, HD 189733b, HD 209458b, TrES-1b and TrES-2b as small crosses. Here, $r$ is calculated as the ratio of the observed value of $R_{\mathrm{p}}\left(M_{\mathrm{p}}, d\right)$ and the value for $R_{\mathrm{p}}\left(M_{\mathrm{p}}, d=\infty\right)$ calculated according to Eq. (A.1). As the mass of all transiting planets lies between $0.11 M_{\mathrm{J}} \leq M_{\mathrm{p}} \leq 3.0 M_{\mathrm{J}}$, one should expect to find all crosses between the two limiting curves. For most planets, this is indeed the case: only for HD 209458b, $r$ is considerably outside the area delimited by the two curves. Different explanations have been put forward for the anomalously large radius of this planet, but so far no conclusive answer to this question has been found (see e.g. Guillot et al. 2006, and references therein). As numerical models cannot reproduce the observed radius of this planet, one cannot expect our approach (which is based on a fit to numerical results) to reproduce it either. For the other planets, Fig. A.1 shows that our approach is a valid approximation.

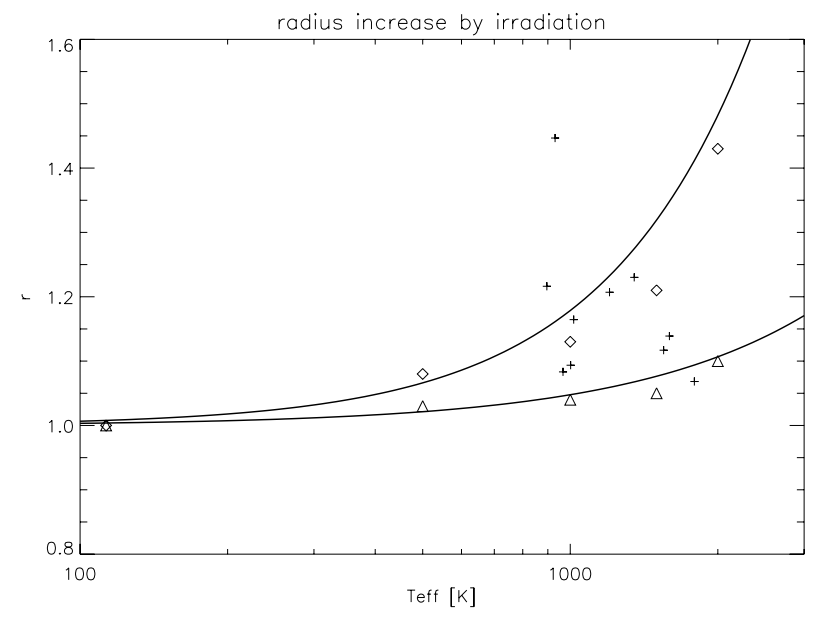

Fig. A.1. Radius increase $r$ as a function of the equilibrium temperature $T_{\mathrm{eq}}$ according to Eqs. (A.5)-(A.7). Upper line: radius increase $r$ due to irradiation for planets of mass $M_{\mathrm{p}}=0.11 M_{\mathrm{J}}$. Lower line: radius increase $r$ due to irradiation for planets of mass $M_{\mathrm{p}}=3.0 M_{\mathrm{J}}$. Open symbols: results of the numerical calculation of Bodenheimer et al. (2003) for planetary masses $M_{\mathrm{p}}=0.11 M_{\mathrm{J}}$ (diamonds) and $M_{\mathrm{p}}=3.0 M_{\mathrm{J}}$ (triangles). Crosses: radius increase for observed transiting planets (see text).

\section{Appendix B: Tidal locking?}

For the estimation of the planetary magnetic dipole moment in Sect. 3.4, we require the planetary rotation rate. This rotation rate rotation greatly depends on whether the planet can be considered as tidally locked, as freely rotating, or as potentially locked. In this appendix, we discuss how we evaluate the tidal locking timescale $\tau_{\text {sync }}$, which decides to which of these categories a planet belongs.

The value of the planetary rotation $\omega$ depends on its distance from the central star. For close-in planets, the planetary rotation rate is reduced by tidal dissipation. In this case, tidal interaction gradually slows down the planetary rotation from its initial value $\omega_{\mathrm{i}}$ until it reaches the final value $\omega_{\mathrm{f}}$ after tidal locking is completed.

It should be noted that for planets in eccentric orbits, tidal interaction does not lead to the synchronisation of the planetary rotation with the orbital period. Instead, the rotation period also depends on the orbital eccentricity. At the same time, the timescale to reach this equilibrium rotation rate is reduced (Laskar \& Correia 2004). Similarly, for planets in an oblique orbit, the equilibrium rotation period is modified (Levrard et al. 2007). However, the locking of a hot Jupiter in a non-synchronous spinorbit resonance appears to be unlikely for distances $\leq 0.1 \mathrm{AU}$ (Levrard et al. 2007). For this reason, we will calculate the timescale for tidal locking under the assumptions of circular orbits and zero obliquity. In the following, the tidal locking timescale for reaching $\omega_{\mathrm{f}}$ is calculated under the following simplifying assumptions: prograde orbit, spin parallel to orbit (i.e. zero obliquity), and zero eccentricity (Murray \& Dermott 1999, Chap. 4).

The rate of change of the planetary rotation velocity $\omega$ for a planet with a mass of $M_{\mathrm{p}}$ and radius of $R_{\mathrm{p}}$ around a star of mass $M_{\star}$ is given by (Goldreich \& Soter 1966; Murray \& Dermott 1999):

$\frac{\mathrm{d} \omega}{\mathrm{d} t}=\frac{9}{4} \frac{1}{\alpha Q_{\mathrm{p}}^{\prime}}\left(\frac{G M_{\mathrm{p}}}{R_{\mathrm{p}}^{3}}\right)\left(\frac{M_{\star}}{M_{\mathrm{p}}}\right)^{2}\left(\frac{R_{\mathrm{p}}}{d}\right)^{6}$, 
where the constant $\alpha$ depends on the internal mass distribution within the planet. It is defined by $\alpha=I /\left(M_{\mathrm{p}} R_{\mathrm{p}}^{2}\right)$, where $I$ is the planetary moment of inertia. For a sphere of homogeneous density, $\alpha$ is equal to $2 / 5$. For planets, generally $\alpha \leq 2 / 5$. $Q_{\mathrm{p}}^{\prime}$ is the modified $Q$-value of the planet. It can be expressed as (Murray \& Dermott 1999)

$Q_{\mathrm{p}}^{\prime}=\frac{3 Q_{\mathrm{p}}}{2 k_{2, \mathrm{p}}}$

where $k_{2, \mathrm{p}}$ is the Love number of the planet. $Q_{\mathrm{p}}$ is the planetary tidal dissipation factor (the larger it is, the smaller is the tidal dissipation), defined by MacDonald (1964) and Goldreich \& Soter (1966).

The time scale for tidal locking is obtained by a comparison of the planetary angular velocity and its rate of change:

$\tau_{\text {sync }}=\frac{\omega_{\mathrm{i}}-\omega_{\mathrm{f}}}{\dot{\omega}}$.

A planet with angular velocity $\omega_{\mathrm{i}}$ at $t=0$ (i.e. after formation) will gradually lose angular momentum, until the angular velocity reaches $\omega_{\mathrm{f}}$ at $t=\tau_{\text {sync }}$. Insertion of Eq. (B.1) into Eq. (B.3) yields the following expression for $\tau_{\text {sync }}$ :

$\tau_{\text {sync }} \approx \frac{4}{9} \alpha Q_{\mathrm{p}}^{\prime}\left(\frac{R_{\mathrm{p}}^{3}}{G M_{\mathrm{p}}}\right)\left(\omega_{\mathrm{i}}-\omega_{\mathrm{f}}\right)\left(\frac{M_{\mathrm{p}}}{M_{\star}}\right)^{2}\left(\frac{d}{R_{\mathrm{p}}}\right)^{6}$.

The importance of this effect strongly depends on the distance $\left(\tau_{\text {sync }} \propto d^{6}\right)$. Thus, a planet in a close-in orbit $(d \lesssim 0.1 \mathrm{AU})$ around its central star is subject to strong tidal interaction, leading to gravitational locking on a very short timescale.

In the following, we briefly describe the parameters $\left(\alpha, Q_{\mathrm{p}}^{\prime}\right.$, $\omega_{\mathrm{i}}$ and $\omega_{\mathrm{f}}$ ) required to calculate the timescale for tidal locking of Hot Jupiters.

Structure parameter $\alpha$ For large gaseous planets, the equation of state can be approximated by a polytrope of index $\kappa=1$. In that case, the structure parameter $\alpha$ (defined by $\alpha=I / M_{\mathrm{p}} R_{\mathrm{p}}^{2}$ ) is given by $\alpha=0.26$ (Gu et al. 2003).
Tidal dissipation factor $Q_{\mathrm{p}}^{\prime}$ For planets with masses of the order of one Jupiter mass, one finds that $k_{2, \mathrm{p}}$ has a value of $k_{2, \mathrm{p}} \approx 0.5$ for Jupiter (Murray \& Dermott 1999; Laskar \& Correia 2004) and $k_{2, \mathrm{p}} \approx 0.3$ for Saturn (Peale 1999; Laskar \& Correia 2004). The value of $k_{2, \mathrm{p}}=0.5$ will be used in this work. With Eq. (B.2), this results in $Q_{\mathrm{p}}^{\prime} \approx 3 Q_{\mathrm{p}}$.

For Jupiter, one finds the following range of allowed values: $6.6 \times 10^{4} \lesssim Q_{\mathrm{p}} \lesssim 2.0 \times 10^{6}$ (Peale 1999). Several estimations of the turbulent dissipation within Jupiter yield $Q_{\mathrm{p}}$-values larger than this upper limit, while other theories predict values consistent with this upper limit (Marcy et al. 1997; Peale 1999, and references therein). This demonstrates that the origin of the value of $Q_{\mathrm{p}}$ is not well understood even for Jupiter (Marcy et al. 1997).

Extrasolar giant planets are subject to strongly different conditions, and it is difficult to constrain $Q_{\mathrm{p}}$. Typically, Hot Jupiters are assumed to behave similarly to Jupiter, and values in the range $1.0 \times 10^{5} \leq Q_{\mathrm{p}}^{\prime} \leq 1.0 \times 10^{6}$ are used. In the following, we will distinguish three different regimes: close planets (which are tidally locked), distant planets (which are freely rotating), and planets at intermediate distances (which are potentially tidally locked). The borders between the "tidally locked" and the "potentially locked" regime is calculated by setting $\tau_{\text {sync }}=100 \mathrm{Myr}$ and $Q_{\mathrm{p}}^{\prime}=10^{6}$. The border between the "potentially locked" and the "freely rotating" regime is calculated by setting $\tau_{\text {sync }}=10$ Gyr and $Q_{\mathrm{p}}^{\prime}=10^{5}$. Thus, the area of "potentially locked" planets is increased.

Initial rotation rate $\omega_{\mathrm{i}}$ The initial rotation rate $\omega_{\mathrm{i}}$ is not well constrained by planetary formation theories. The relation between the planetary angular momentum density and planetary mass observed in the solar system (MacDonald 1964) suggests a primordial rotation period of the order of $10 \mathrm{~h}$ (Hubbard 1984, Chap. 4). We assume the initial rotation rate to be equal to the current rotation rate of Jupiter (i.e. $\omega=\omega_{\mathrm{i}}=\omega_{\mathrm{J}}$ ) with $\omega_{\mathrm{J}}=1.77 \times 10^{-4} \mathrm{~s}^{-1}$.

Final rotation rate $\omega_{\mathrm{f}}$ As far as Eq. (B.4) is concerned, $\omega_{\mathrm{f}}$ can be neglected (Grießmeier 2006). 